\title{
Exploring many-body theories in light nuclei
}

\author{
Document Version \\ Accepted author manuscript
}

Link to publication record in Manchester Research Explorer

\section{Citation for published version (APA):}

Bishop, RF., Flynn, MF., Boscá, MC., Buendĺa, E., \& Guardiola, R. (1990). Exploring many-body theories in light nuclei. In W. Greiner, \& H. Stõcker (Eds.), The Nuclear Equation of State, Part A: Discovery of Nuclear Shock Waves and the EOS (pp. 605-614). Plenum Publishing Corporation.

http://personalpages.manchester.ac.uk/staff/raymond.bishop/RFB_papers/[078] NuclEqState_A(1990)605

Published in:

The Nuclear Equation of State, Part A

\section{Citing this paper}

Please note that where the full-text provided on Manchester Research Explorer is the Author Accepted Manuscript or Proof version this may differ from the final Published version. If citing, it is advised that you check and use the publisher's definitive version.

\section{General rights}

Copyright and moral rights for the publications made accessible in the Research Explorer are retained by the authors and/or other copyright owners and it is a condition of accessing publications that users recognise and abide by the legal requirements associated with these rights.

\section{Takedown policy}

If you believe that this document breaches copyright please refer to the University of Manchester's Takedown Procedures [http://man.ac.uk/04Y6Bo] or contact uml.scholarlycommunications@manchester.ac.uk providing relevant details, so we can investigate your claim.

\section{open 2 access}




\title{
EXPLORING MANY-BODY THEORIES IN LIGHT NUCLEI
}

\author{
R.F. Bishop and M.F. Flynn \\ Department of Mathematics, UMIST \\ P.O.Box 88,Manchester M60 1QD England \\ and \\ M.C. Boscá, E. Buendía and R. Guardiola \\ Departamento de Física Moderna, Universidad de Granada \\ 18071 Granada, Spain
}

\begin{abstract}
A brief review of several many-body theories is presented with applications to the ${ }^{4} \mathrm{He}$ nucleus in a simplified model which only considers Wigner type nucleon-nucleon interactions. Exact results are obtained with the Diffusion Monte Carlo method, and the results from the Jastrow variational method as well as linearized version of coupled cluster theory are compared with them.
\end{abstract}

\section{Introduction}

The Quantum Many-Body Problem presents us with a simply-stated but otherwise quite formidable task, namely the determination of the ground state (and eventually also the excitations) of a system of many mutually interacting particles, in the limit as this number of particles grows to infinity at a constant density. The actual solution of the many-body Schrödinger equation is moreover complicated by the strong coupling character of the usual two-body interactions, which thereby prevents the use of naive perturbative methods. The time-honored Brueckner-Bethe-Goldstone (BBG) theory was invented as a special perturbation expansion technique to solve this problem. In addition, the formalism has a deep physical content.

The optimistic belief at the beginning of the seventies [1] was that BBG theory gave the appropriate tools to solve the many-body problem, at least in nuclear matter, but soon some variational calculations of Jastrow type showed the deficiencies of lowest-order BBG theory. This was called the crisis in nuclear matter theory (see [2] for a concise but precise historical review) and during the last decade several new many-body theories were developed and brought into use. Here we will refer to three of them, namely the Green Function Monte Carlo method [3], the hypernetted chain HNC/FHNC massive summation scheme for the 
Jastrow variational description [4] and the $\exp (S)$ method or Coupled Cluster theory [5]. A resolution of the crisis was illustrated during the first conference on Recent Progress in Many-Body Theories held in Trieste in 1978 [6].

Unfortunately, the plettora of technicalities required for the application of these manybody theories to infinite systems, obscure their underlying simplicity. Here we will attempt a brief description of these theories through a simpler yet still very challenging problem, with a basic didactical objective. We thus concentrate on the study of the ${ }^{4} \mathrm{He}$ nucleus, and all complicated questions regarding discrete degrees of freedom (spin and isospin) will be absent by limiting ourselves to Wigner-type nucleon-nucleon interactions. Because of the absence of spin/isospin degrees of freedom in the hamiltonian the four nucleons may be considered as distinguishable, or, in other words, we will deal effectively with a system of four bosons. Thus another of the technical complications will be absent, namely antisymmetrization.

Given that our objective is to show the usefulness of many body theories in a wide range of physical situations, we will nevertheless consider four kinds of nuclear interactions, ranging from mild two-body potentials to realistic interactions with a strongly repulsive core at short distances. We describe them below in order of increasing complexity.

The simplest interaction considered was introduced by Kalos [7] in a pioneering work on Green Function Monte Carlo method. The aim of that work was the determination of the coupling constant of a fixed-shape two-body potential which would produce the experimental binding energy of $28.3 \mathrm{MeV}$ of ${ }^{4} \mathrm{He}$, i.e. an inverse many-body problem. The interaction is purely attractive and has gaussian shape. Next we consider the well known Brink Boeker BB1 potential [8], which is an effective interaction with parameters adjusted so as to obtain the proper saturation conditions in ${ }^{4} \mathrm{He},{ }^{16} \mathrm{O}$ and nuclear matter when using uncorrelated wave functions. This interaction has been widely used in spectroscopic calculations, and it will certainly overbind ${ }^{4} \mathrm{He}$ when used as a bare microscopic interaction, as considered here. Finally we have considered two realistic interactions known as S3 [9] and MTV [10]. These two interactions are only defined in the $\ell=0$ channel, so that the word realistic should be considered somewhat sceptically in so far as the interactions do not contain spin-orbit nor tensor components. We will use these interactions as local potentials acting in all partial waves. The S3 and MTV interactions are of particular interest because of the large amount of work devoted to them in the study of three- and four-nucleon systems.

As said above, we have only considered the Wigner part of the above interactions, so that we will actually obtain upper bounds to the ground state (g.s.) energy corresponding to the full interactions. The interactions will be referred to respectively by the letters $\mathrm{K}$, $\mathrm{B}, \mathrm{S}$ and $\mathrm{M}$, or sometimes, in the case of the latter three, by the more usual acronyms BB1, S3 and MTV. Potentials K, B and S are a combination of gaussians, and this fact is particularly useful because all of the required matrix elements can be computed by means of semi-numerical algorithms, which both considerably reduce the calculational time and avoid unpleasant cumulative numerical errors. This will permit us to carry out calculations in a very large configuration space. The MTV potential, on the other hand, is a combination of yukawians.

The shape of these four interactions is shown in Figure 1, which also illustrates their various degrees of difficulty to handle. This is basically related to the importance of the short range repulsion. 


\section{The diffusion Monte Carlo method}

Stochastic methods can be used to integrate the many body Schrödinger equation exactly in the simplified case of boson systems. These methods are known as Green Function Monte Carlo [3] and Diffusion Monte Carlo [11,12,13]. We will consider only the latter and will refer to it by the acronym DFMC.

Three are three basic ingredients for DFMC theory. First is the consideration of the time-dependent Schrödinger equation in terms of imaginary time. Starting from an initial state $\Psi(0)$ its imaginary-time evolution is governed by a linear combination of exponentially increasing or decreasing functions, instead of the normal oscillatory evolution of amplitudes in real time. Moreover, if the hamiltonian is shifted by an amount $\mathcal{E}$ equal to the g.s. energy $E_{g s}$, all the amplitudes will drop to 0 at $t \rightarrow \infty$ with the exception of the g.s. component, provided that $\Psi(0)$ has a nonzero projection on the exact ground state. In other words, the norm of $\Psi(t)$ will stabilize when $\mathcal{E}=E_{g s}$ at $t \rightarrow \infty$. This is a way of determining $E_{g s}$ known as the grow estimator.

The imaginary-time equation is solved with the help of the time-dependent Green function, in such a form that the equation

$$
\Psi\left(\mathcal{R}, t_{0}+t\right)=\int d \mathcal{R}^{\prime} G\left(\mathcal{R}, \mathcal{R}^{\prime}, t\right) \Psi\left(\mathcal{R}^{\prime}, t_{0}\right)
$$

gives the wave function after a time step $t$. In this and subsequent equations $\mathcal{R}$ will represent the set of all coordinates of the many body system. The time-dependent and energy-shifted Green function is given by the matrix element

$$
G\left(\mathcal{R}, \mathcal{R}^{\prime}, t\right)=\left\langle\mathcal{R}|\exp [-(H-\mathcal{E}) t]| \mathcal{R}^{\prime}\right\rangle
$$

Certainly, the determination of the Green function is a very formidable problem, which is actually more difficult than determining only the ground state. It is for this reason that one introduces the second basic ingredient of DFMC theory. One may approximate $G\left(\mathcal{R}, \mathcal{R}^{\prime}, t\right)$ for a sufficiently small value of $t=\tau$ by the form (see [14] for a detailed derivation)

$$
G\left(\mathcal{R}, \mathcal{R}^{\prime}, \tau\right)=\frac{\exp \left\{-\left[\mathcal{R}-\mathcal{R}^{\prime}\right]^{2} / 4 D \tau\right\}}{(4 \pi D \tau)^{3 A / 2}} \exp \left[\left(\mathcal{E}-\left(V(\mathcal{R})+V\left(\mathcal{R}^{\prime}\right)\right) / 2\right) \tau\right]
$$

In this equation $D=\hbar^{2} / 2 m$ is called the diffusion constant, $A$ is the number of particles and $V(\mathcal{R})=\sum_{i<j} V\left(r_{i j}\right)$ is the full interaction potential.

Given that we want the $t \rightarrow \infty$ limit, eq. (1) is applied repeatedly to an initial $t=0$ state using the approximate form eq. (3) up to a sufficiently large time. In parallel, $\mathcal{E}$ is adjusted to stabilize the norm, so that we obtain in this manner the ground-state energy.

We arrive now to the third basic ingredient of DFMC theory, which is the way in which the wave function is represented. This hinges on the special property of the positivity of both the (bosonic) ground-state wave function and the small time Green function. Because of this one can interpret $\Psi(t)$ as a probability distribution function and, in turn, represent $\Psi(t)$ by a set of $3 A$ dimensional random vectors. In normal Monte Carlo practice one knows the functional form of the distribution function and the task is to get random numbers corresponding to this distribution. Here one works in the inverse way: the distribution function is not known but it is represented by a set of random points. 


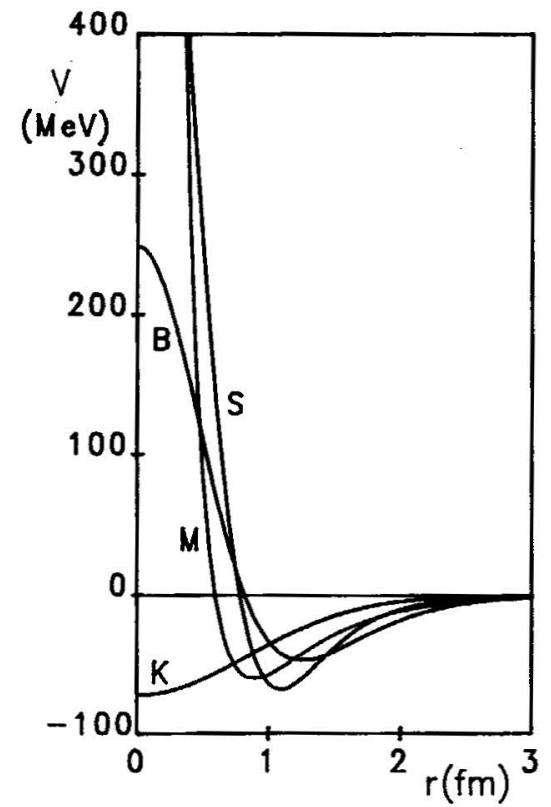

Figure 1. The radial dependence of the two body interactions

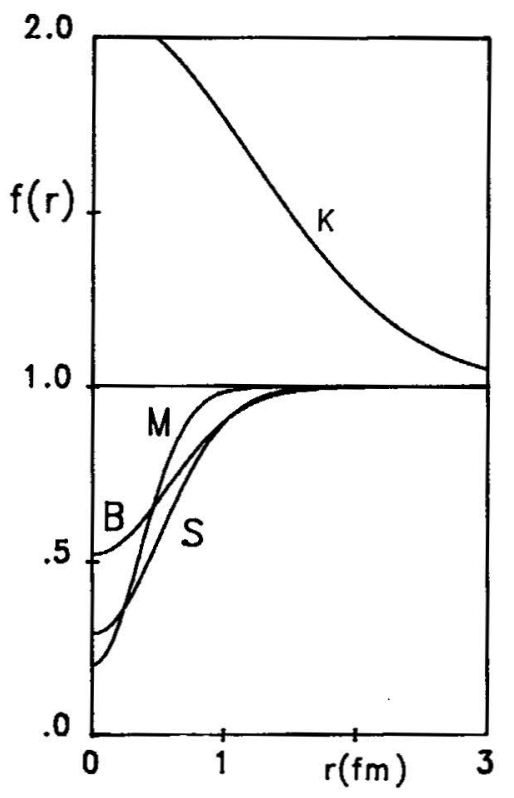

Figure 3. Radial dependence of the correlation factors corresponding to the four interactions

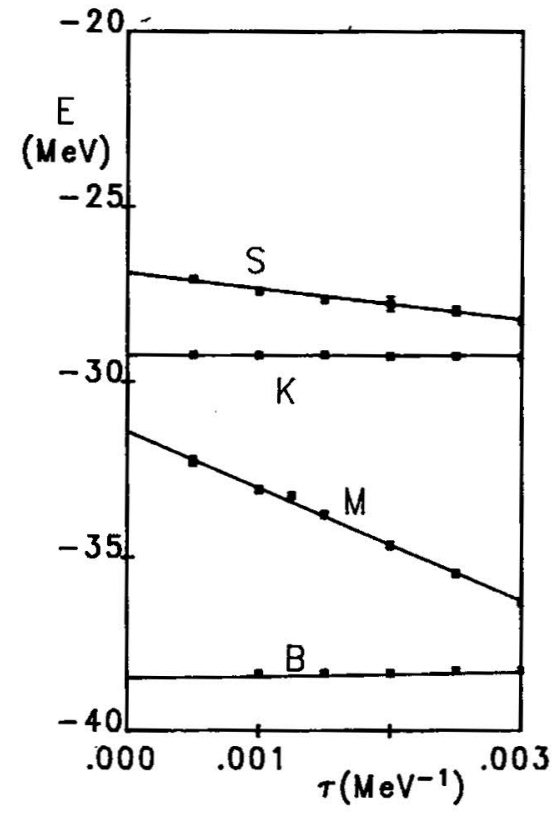

Figure 2. The variation of the sampled energy as a function of the time step

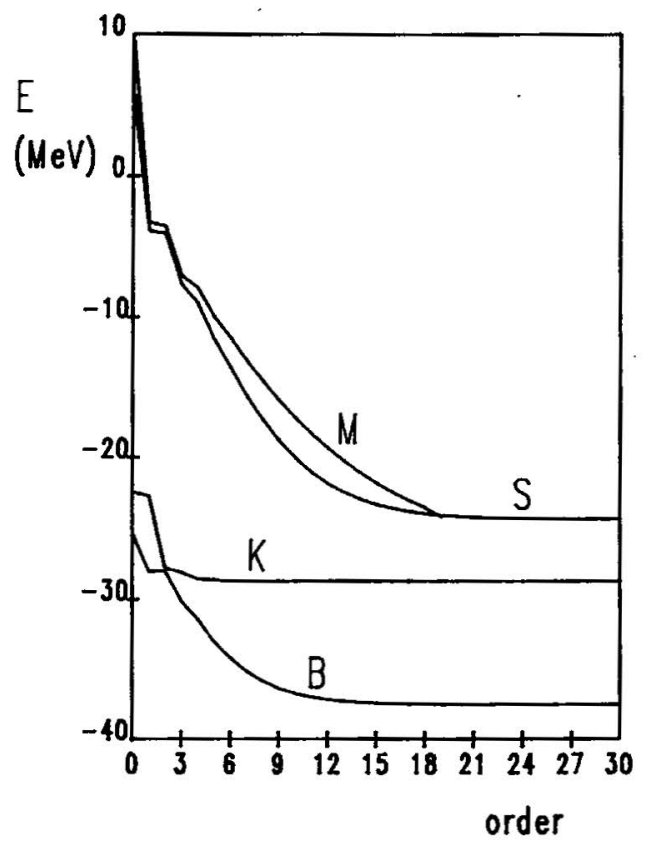

Figure 4. The convergence of linear CC calculations as a function of the number of basis states 
With this representation of $\Psi(t)$ in mind, the action of eq. (1) is to obtain a new set of random vectors corresponding to $t+\tau$ from the previous set at time $t$, and the rules of this evolution are contained in eq. (3). The first term of eq. (3) is a normalized gaussian and corresponds to an isotropic diffusion centered at $\mathcal{R}^{\prime}$. The second term is an unnormalized exponential and corresponds to a source or sink of points. By adjusting $\mathcal{E}$ to the g.s. energy, the average population remains stable, in correspondence with the stability of the norm of the wave function.

There are still three technical points to be mentioned. First, the results must be extrapolated to $\tau \rightarrow 0$, and several runs corresponding to different values of $r$ must be carried out. Second, one has to distinguish the first set of time steps, which serves to stabilize $\mathcal{E}$ from another subsequent set of steps to control the statistical error of the result. Finally we should mention a very important improvement of the previous description known as importance sampling, which incorporates in the algorithm all information corresponding to an assumed good trial function, and has the effect of accelerating the convergence and lowering the statistical uncertainty of the result $[12,13]$.

Our results are presented in Figure 2 for the four interactions and several values of the time step $\tau$. In all cases we have found a linear dependence with the time step, and the intercept of these straight lines with the vertical axis gives the exact (within statistical errors) value of the ground state energy of ${ }^{4} \mathrm{He}$ for the mentioned interactions. We have used the Jastrow function to be described in the next section as the importance sampling function, and a total of 900.000 points was used to sample each point of Figure 2.

The numerical values of the energy are shown in the last row of Table 1 . Our result for the MTV potential is compatible with the value of $-31.3 \pm 0.2 \mathrm{MeV}$ obtained by Kalos and Zabolitzky [15]. Our result for the Kalos potential is $1 \mathrm{MeV}$ lower than the value of $-28.3 \mathrm{MeV}$ obtained in the old paper of Kalos [3]. On the other hand the statistical error in Ref. [3] is surely quite high, and our value is certainly in full agreement with the best variational estimates.

\section{Jastrow variational method}

The so-called Jastrow trial function was introduced as early as 1940 by Bijl [16] and afterwards by Dingle [17] and Jastrow [18]. One may view this theory as a kit to taylor the variational wave function in such a way that physical requirements related to the nature of the system or to the properties of the two body interaction are manually incorporated into the trial function.

Consider our simple problem. Since we deal with a system of finite size, we may take a so a shell model-like wave function which localizes all particles around the center of mass. Second, we have in some cases interactions which are strongly repulsive at short distances so that the wave function should be very small or even null when $r_{i j} \rightarrow 0$ for any pair $(i j)$ of particles. Moreover one would not like to spoil the independent particle motion when one of the particles moves far away from the rest. To fulfill these last two characteristics we may put for each pair of particles a Jastrow correlation factor $f\left(r_{i j}\right)$ which is small when $r_{i j} \rightarrow 0$ 
Table 1. The ground-state energy of ${ }^{4} \mathrm{He}$ computed with various many body techniques

\begin{tabular}{|c|c|c|c|c|}
\hline METHOD & KALOS & BB1 & S3 & MTV \\
\hline HOWF & -23.15 & -28.16 & -5.89 & -6.40 \\
\hline HOWF+J & -29.11 & -36.44 & -24.29 & -29.48 \\
\hline EXP+J & $-27.3 \pm 0.2$ & $-37.6 \pm 0.2$ & $-26.0 \pm 0.2$ & $-30.2 \pm 0.3$ \\
\hline CC2-L & -28.74 & -37.80 & -25.31 & -24.17 \\
\hline DFMC & $-29.25 \pm 0.05$ & $-38.5 \pm 0.1$ & $-26.9 \pm 0.2$ & $-31.5 \pm 0.2$ \\
\hline
\end{tabular}

and goes to a constant, e.g. 1, when $r_{i j} \rightarrow \infty$. Thus, the Jastrow variational function for our problem is

$$
\Psi_{J}=\prod_{i<j} f\left(r_{i j}\right) \prod_{i} \phi\left(r_{i}\right)
$$

In more complex systems one may also introduce such other properties as statistics, angular momentum or isospin coupling, spin- and isospin-dependent correlations, triplet correlations and so on. All of these properties are incorporated into the trial wave function in the same way as above.

In eq. (4) there appear single-particle wave functions referred to the origin of coordinates, so that $\Psi_{J}$ is not translationally invariant. The obvious way of restoring translational invariance is to consider that all $r_{i}$ appearing in eq. (4) are actually distances from the center of mass of the system. However, this will create serious technical problems when computing the expectation value of the hamiltonian. Another way of dealing with this question is to consider single particle wave functions which are eigenstates of the harmonic oscillator potential (HOWF). Then $\Psi_{J}$ factorizes into two terms, one of which depends only on the center of mass coordinate and the other being translationally invariant. Then, the true energy of the system will be the difference of the energy computed with the function $\Psi_{J}$ and the kinetic energy of the center of mass. This is the procedure that we will follow. Note that the factorization property comes from the shell model part of the wave function, and this property will be preserved in so far as such additional factors, as the Jastrow factor $f\left(r_{i j}\right)$, depend only on relative distances.

The most straightforward calculation with $\Psi_{J}$ consists in assuming functional forms for $f$ and $\phi$ which depend on several parameters and then searching for the values of these parameters which give a minimum for the expectation value of the hamiltonian. We have assumed the very simple forms

$$
\begin{aligned}
& \phi(r)=\exp \left(-\frac{1}{2} \alpha^{2} r^{2}\right) \\
& f(r)=1+a \exp \left[-(r / b)^{2}\right]
\end{aligned}
$$

which have three free parameters: $\alpha$, which controls the size of the nucleus, and $a$ and $b$ which correspond to the depth and range of the correlation. The value of the depth $a$ is mainly determined by the nature of the interaction alone. However, $\alpha$ and $b$ are strongly correlated so that one cannot search for the position of the minimum by considering independent variations of these parameters. 
The energies obtained by means of this simple variational function are shown again in Table 1. The row labelled HOWF corresponds to the expectation value in the absence of correlations, and the row labelled HOWF $+\mathrm{J}$ incorporates the two body correlations in the way above described. Note that the values of the harmonic oscillator parameter $\alpha$ for these two rows are not the same. It is very satisfying to realize that even with so simple an ansatz for the wave function one obtains almost the entire binding energy of the system, being only $2 \mathrm{MeV}$ above the exact DFMC result except for the Kalos potential, in which case our results are almost exact. Part of this energy difference is certainly related to the very sharp shape of the nuclear surface produced by the harmonic oscillator central field. If in eq. (5) we replace $\phi(r) \rightarrow 1$ and at the same time multiply $f(r)$ by $\exp (-r / c)$ we gain 1 or $1.5 \mathrm{MeV}$, with the exception of $\mathrm{K}$ interaction which prefers a well defined surface. The values corresponding to this calculation are shown in the row labelled EXP $+J$ in Table 1. Note that all integrals have been computed with the Monte Carlo method, and the errors appearing in Table 1 are the statistical errors of the computation.

The gain in energy due to Jastrow correlations is impressive, ranging from $6 \mathrm{MeV}$ for the Kalos potential up to more than $20 \mathrm{MeV}$ for the S3 and MTV potentials. The flexibility of this simple form of the correlation is also noticeable, being able to adapt itself to this wide range of interactions. Figure 3 shows the shape of the correlation of Eq. (5) for the four potentials considered, showing a clear relationship between the interaction and $f$.

We have thus seen how simple it is to obtain up to $90 \%$ or even $95 \%$ of the binding energy. A natural question arises: How far may we go with a Jastrow ansatz using only two-body correlations?. To answer this question one has to compute the energy with a wave function like eq. (4) but allowing the maximum freedom in the variational determination of $f$. In other words, one has to carry out an Euler-Lagrange calculation with respect to f. Calculations of this kind for the MTV potential have been carried out at Urbana [19] by solving an Euler-Lagrange equation for en energy functional obtained at second order of the cluster expansion with the result of $-31.19 \pm 0.05 \mathrm{MeV}$, and at Pisa [20] using the exact functional, to obtain $-31.35 \mathrm{MeV}$. This small gain of $1 \mathrm{MeV}$ is very costly to obtain. Comparing these numbers with the DFMC results one may conclude that the Jastrow form describes almost completely the physical system.

\section{Coupled cluster theory}

$\operatorname{Exp}(S)$ or Coupled Cluster (CC) theory was first proposed by Coester and Kümmel [21]. Its most important feature is the incorporation from the very outset of Goldstone's linked cluster theorem. A full review of the theory may be found in [5]. The exact wave function is written in the following form. Assume a filled Fermi sea, and a series of one-body $S_{1}$, two-body $S_{2}$, three-body $S_{3}, \ldots$ operators which correspond to coherent $1 \mathrm{p}-1 \mathrm{~h}, 2 \mathrm{p}-2 \mathrm{~h}$, $3 \mathrm{p}-3 \mathrm{~h}$... excitations, respectively. Then the ansatz for the ground state wave function is

$$
\Psi_{C C}=\exp \left[S_{1}+S_{2}+S_{3}+\ldots\right] \Psi_{0}
$$

with $\Psi_{0}$ corresponding to the Fermi sea. Expanding in powers the above exponential one may understand the physical content of the theory. For example, to produce $3 \mathrm{p}-3 \mathrm{~h}$ excitations 
one has three possibilities, represented by the operators $S_{1}^{3}, S_{1} S_{2}$ and $S_{3}$. We then realize that $S_{3}$ will only contain true linked $3 \mathrm{p}-3 \mathrm{~h}$ excitations, the unlinked ones being contained in the other two operator products.

In the form given by eq. (6) the theory is not translationally invariant (in finite systems), because the exponential factor spoils the basic factorizability property of the harmonic oscillator $\Psi_{0}$ wave function. For a system of bosons we have found [22] a simple modification of eq. (6) which preserves the translational invariance. Up to two body clusters it reads

$$
\Psi_{C C 2}=N\left[\exp \mathcal{S}_{2}\right] \Psi_{0}
$$

where $N$ represents the normal ordering, and $\mathcal{S}_{2}$ is an admixture of the previous $S_{1}$ and $S_{2}$ given by

$$
\mathcal{S}_{2}=\sum_{n=1}^{\infty} S_{2}^{n} \Omega_{2}^{n}
$$

with

$$
\Omega_{2}^{n}=\sum_{n_{i} \ell_{i} n_{j}}\left\langle n 0,00,0 \mid n_{i} \ell_{i}, n_{j} \ell_{i}, 0\right\rangle\left[a_{n_{i} \ell_{i}}^{\dagger} \times a_{n_{j} \ell_{i}}^{\dagger}\right]^{0} a_{0} a_{0}
$$

where the coefficients $S_{2}^{n}$ are numerical amplitudes to be determined, $a_{n \ell m}^{\dagger}$ creates a singleparticle harmonic oscillator state with quantum numbers $(n \ell m)$, the subscript $0 \equiv(000)$, and the sum over $n_{i}, \ell_{i}$ and $n_{j}$ has no other restrictions than those implied by the BrodyMoshinsky bracket. In particular, $\left(n_{i} \ell_{i}\right)$ may be equal to (00) and eq. (8) contains both $1 \mathrm{p}-1 \mathrm{~h}$ and $2 \mathrm{p}-2 \mathrm{~h}$ excitations. The particular combination shown will guarantee translation invariance. Note that $\Omega_{2}^{n}$ corresponds globally to a excitation energy of $2 n \hbar \omega$.

Perhaps it is more interesting to look at the coordinate representation of the action of $\mathcal{S}_{2}$ on the vacuum state. Using standard Fock algebra one may obtain the correspondence,

$$
\Omega_{2} \Psi_{0}=\text { Const } \times \sum_{i<j} L_{n}^{(1 / 2)}\left(\alpha^{2} r_{i j}^{2} / 2\right) \Psi_{0}
$$

where $L_{n}^{(1 / 2)}$ is the associated Laguerre polynomial corresponding to an $(n, \ell=0)$ relative motion of a pair of nucleons. Quadratic terms like $\Omega_{2}^{n} \Omega_{2}^{m}$ correspond to one pair $(i j)$ in relative state $(n 0)$ and another pair $(k l)$ in relative state $(m 0)$ with both particles $k$ and $l$ different from $i$ and $j$.

Restricting ourselves to linear terms only from the exponential in eq. (7), we may rewrite the simplified CC translationally invariant wave function in the general coordinate form

$$
\Psi_{C C 2-L}=\left[1+\sum_{i<j} g\left(r_{i j}\right)\right] \Psi_{0}
$$

to bring out the similarity with the Jastrow ansatz. One can then work with eq. (8) using standard shell model and Fock algebra machinery, or with equation (11) in the same manner as in the case of Jastrow form. We have preferred the first form, to maintain ourselves as close to the original ansatz as possible, but for the case of gaussian shaped interactions a special algorithm based on the properties of the generating function of Laguerre polynomials was used, allowing us to extend the calculation up to incredibly high values of $n \simeq 30$, or equivalently up to $60 \hbar \omega$ excitation quanta. Solving the linear CC equations in the usual 
form [5] is equivalent to diagonalize a finite matrix, so that the linear CC method gives actually a variational upper bound to the binding energy. The variation of the results with the number of excitations $n$ is shown in Figure 4, and the convergence is strongly tied to the complexity of the interaction, being very rapid for the Kalos potential and quite slow for the S3 interaction. The results for the MTV potential were obtained by using the standard shell-model machinery and we could not go further than $n=19$. The energy at this value is not yet stabilized. This simply means that we have used a poor basis in which to solve CC equations, which would perhaps have been better solved in coordinate representation [23].

The numerical results of the binding energy are shown again in Table 1, in the row labelled CC2-L ( $\mathrm{L}$ for linear). Note that the value for MTV is still far from convergence. It was a nice surprise to find that the CC2-L results, corresponding to an ansatz which is much simpler than the Jastrow variational method, produced results of comparable quality. Unfortunately, the only case with which we may compare corresponds to the MTV potential, where we have not attained convergence. For this interaction Zabolitzky [24] obtained the value of $-31.24 \mathrm{MeV}$, very close to the exact DFMC result, by using the full CC theory. There is also a configuration interaction calculation [25] for MTV potential, which considers all possible $\mathrm{p}-\mathrm{h}$ excitations up to $10 \hbar \omega$ (a total of 2765 basis states) with the result of $-18.31 \mathrm{MeV}$, which is very far from both our $n=19$ result ( 20 basis states) and, obviously, from the fully converged value. We could say that $\mathrm{CC}$ theory also gives a rather clever selection of the important basis states.

\section{Summary}

This work was planned with a basic didactical purpose. The hope is that after reading it, the reader will be convinced of the basic simplicity of the theories discussed here, as well as of their high quality, even with the drastic simplifications and simple forms used for the calculations. Nevertheless, we should not conceal the fact that even with all these simplifications the calculations still involve a large amount of algebraic as well as computational effort. Certainly, the many-body problem is not a simple question.

\section{Acknowledgements}

This work was developed under an Acciones Integradas program between Spain and the United Kingdom. The authors acknowledge the financial support from the corresponding joint committee. M.C.B., E.B. and R.G. are also supported by the Comision Interministerial de Ciencia y Tecnología, Spain under contract 969/87. R.F.B. and M.F.F. also acknowledge the support of a research grant from SERC of Great Britain.

\section{References}

[1] H.A. Bethe, Ann. Rev. Nucl. Sci 21 (1971) 93.

[2] J.W. Clark, Prog. Part. Nucl. Phys. 2 (1979) 89. 
[3] D.M. Ceperley and M.H. Kalos, Quantum Many Body Problems in Monte Carlo Methods is Statistical Physics, K. Binder editor (Springer Verlag, New York 1979).

[4] S. Rosati and S. Fantoni, Correlations in Infinite Systems in The Many Body Problem: Jastrow Correlations versus Brueckner Theory, R. Guardiola and J. Ros editors (Springer Verlag, New York 1981), p. 1.

[5] H. Kümmel, K.H. Lührmann and J.G. Zabolitzky, Phys. Rep. 36C (1978) 1.

[6] Proceedings of the First International Conference on Condensed Matter Theories, C. Ciofi degli Atti, A. Kallio and S. Rosati editors. Nucl. Phys. A328 (1979).

[7] M.H. Kalos, Phys. Rev. 128 (1962) 1791.

[8] D.M. Brink and E. Boeker, Nucl. Phys. A91 (1967) 1.

[9] I.R. Afnan and Y.C. Tang, Phys. Rev. 175 (1968) 1337.

[10] R.A. Malfliet and J.A. Tjon Nucl. Phys. A127 (1969) 161.

[11] J.B. Anderson, J. Chem. Phys. 63 (1975) 1499, 65 (1976) 4121 and 73 (1980) 3879.

[12] P.J. Reynolds, D.M. Ceperley, B.J. Alder and W.A. Lester Jr, J. Chem. Phys. 77 (1982) 5593.

[13] R. Guardiola, Monte Carlo Techniques in the Many Body Problem, in First International Course on Condensed Matter, D. Prosperi, S. Rosati and G. Violini editors. (World Scientific, Singapore, 1988).

[14] J. Vrbik and S.M. Rohtstein, J. Comput. Phys. 63 (1986) 130.

[15] M.H. Kalos and J.G. Zabolitzky, Nucl. Phys. A356 (1981) 114.

[16] A. Bijl, Physica 7 (1940) 869.

[17] R.B. Dingle Phil. Mag. 40 (1949) 573.

[18] R. Jastrow, Phys. Rev. 98 (1955) 1479.

[19] J. Carlson and V.R. Pandharipande, Nucl. Phys. A371 (1981) 301.

[20] L. Bracci, S. Rosati and M. Viviani, in Proc. Secondo Convegno su Problemi di Fisica Nucleare Teorica, (ETS Editrice, Pisa 1988), p. 34.

[21] F. Coester and H. Kümmel, Nucl. Phys. 17 (1960) 477.

[22] R.F. Bishop, M.F. Flynn, M.C. Boscá, E. Buendía and R. Guardiola, in preparation.

[23] J.G. Zabolitzky, Nucl. Phys. A228 (1974) 272.

[24] J.G. Zabolitzky, Phys. Lett. 100B (1981) 5.

[25] R. Ceuleneer and P. Vandepeutte, Phys. Rev. C 31 (1985) 1528. 